\title{
Diagnostic value of $P R N D$ gene expression profiles in astrocytomas: Relationship to tumor grades of malignancy
}

\author{
S. COMINCINI ${ }^{1}$, V. FERRARA $^{1}$, A. ARIAS ${ }^{1}$, A. MALOVINI ${ }^{1}$, A. AZZALIN ${ }^{1}$, L. FERRETTI $^{1}$, E. BENERICETTI $^{2}$, \\ M. CARDARELLI ${ }^{3}$, M. GEROSA ${ }^{3}$, M.G. PASSARIN ${ }^{4}$, S. TURAZZI $^{3}$ and R. BELLAZZI ${ }^{5}$ \\ ${ }^{1}$ Dipartimento di Genetica e Microbiologia, Università degli Studi di Pavia, Pavia; ${ }^{2}$ Azienda Ospedaliera - \\ Universitaria di Parma, Parma; ${ }^{3}$ Clinica Neurochirurgica Università di Verona; ${ }^{4}$ U.O. Neurologia, Azienda Ospedaliera - \\ Universitaria di Verona, Verona; ${ }^{5}$ Dipartimento di Informatica e Sistemistica, Università degli Studi di Pavia, Pavia, Italy
}

Received December 14, 2006; Accepted January 24, 2007

\begin{abstract}
Doppel $(P R N D)$ is a paralogue of the mammalian prion $(P R N P)$ gene. It is abundant in testis and, unlike $P R N P$, it is expressed at low levels in the adult central nervous system (CNS). Besides, doppel overexpression correlates with some prion-disease pathological features, such as ataxia and death of cerebellar neurons. Recently, ectopic expression of doppel was found in two different tumor types, specifically in glial and haematological cancers. In order to address clinical important issues, $P R N D$ mRNA expression was investigated in a panel of 111 astrocytoma tissue samples, histologically classified according to the World Health Organization (WHO) criteria (6 grade I pilocytic astrocytomas, 15 grade II low-grade astrocytomas, 26 grade III anaplastic astrocytomas and 64 grade IV glioblastoma multiforme). Real-time $P R N D$ gene expression profiling, after normalisation with $G A P D H$, revealed large differences between low (WHO I and II) and high grade (III and IV) of malignancy $(\mathrm{P}<0.001)$. Extensive differences in $P R N D$ gene expression were also found within each grade of malignancy, suggesting that $P R N D$ mRNA quantitation might be useful to distinguish astrocytoma subtypes, and important in disease stratification and in the assessment of specific treatment strategies.
\end{abstract}

\section{Introduction}

Astrocytomas are the most common CNS neoplasms (1) and are graded I-IV according to their histological features (2). Low-grade astrocytomas typically display a long clinical history with relatively benign prognosis, whereas the prognosis of high-grade astrocytomas is devastating (3). However, low-

Correspondence to: Dr Sergio Comincini, Dipartimento di Genetica e Microbiologia, Università di Pavia, via Ferrata 1, 27100 Pavia, Italy

E-mail: sergio.c@ipvgen.unipv.it

Key words: astrocytoma, gene expression, molecular diagnosis, real-time PCR analysis grade astrocytomas have an inherent tendency to progress toward more malignant forms. This progression has been thought to result from an accumulation of genetic events that leads to the clonal expansion of a malignant cell (4). Some of these events, such as the inactivation of the $p 53$ tumor suppressor gene in the initial steps of carcinogenesis and amplification of the epidermal growth factor receptor $(E G F R)$ gene in the late stages have been identified (reviewed in ref. 5,6). Previous studies have documented patterns of gene expression associated with specific clinical grades of astrocytomas (7-10). In addition, malignant astrocytic gliomas either arise de novo (primary) or from the progression of lower-grade astrocytic gliomas (secondary). The most frequent genetic anomalies found in primary astrocytomas are a gain of chromosome 7 and amplification of EGFR, LOH of chromosome 10, and mutation or deletion of PTEN and p16 tumor suppressor genes (11). Frequent occurrences of mutation of the p53 tumor suppressor gene and alteration of genes involved at specific cell cycle checkpoints have been observed in secondary astrocytoma patients (12).

Although the cellular origin of CNS tumors remains unclear, it has been suggested that they could derive from an undifferentiated resident blast cell (6). CNS tumor cells may exhibit a gene expression pattern similar to that of the cells in the course of CNS development. Thus, the identification of such specific genes is valuable in the CNS tumor diagnosis, particularly if they are expressed only in tumors and not in the corresponding adult non-neoplastic tissue (13). To this regard, the first prion-gene paralogue, doppel (Prnd), has been described in rodents (14). Subsequently, the doppel gene has been confirmed in man, cattle and sheep (15). Comparative gene expression analysis has been reported on different patterns of temporal and spatial expression among prion and doppel. Thus, whereas prion protein $(\mathrm{PrP})$ is widely expressed, showing the highest expression profiles within the CNS, doppel shows barely detectable levels in most tissues (16). Doppel protein (Dpl) was found highly expressed only in adult and fetal testis, and, different groups proposed an involvement in male gametogenesis (reviewed in ref. 17). Based on the structural similarities between PrP and Dpl, a role of doppel in the development of prion neurodegenerative diseases was hypothesised (18). Specifically, when ectopically 
expressed in some $\operatorname{Prnp}^{0 / 0}$ transgenic lines, Dpl causes Purkinje cell death and ataxia (14). However, Dpl-induced neurodegeneration can be rescued by the introduction of a Prnp transgene, suggesting the possibility that the protein interacts or competes with PrP in a sort of molecular antagonismmodel (19). Further studies were performed to demonstrate a direct involvement of doppel in prion-diseases. From these analyses, doppel seems not to be associated with the diseases, neither if one considers the gene variability and expression (15), nor if one examines the role of the protein at the pathological lesions (20). Consequently, the expression of the doppel gene has been investigated in other pathological contexts. Ferrer and collaborators (21) have recently reported a selective Dpl immunoreactivity in dystrophic neuritis in Alzheimer's disease patients. In tumors, the first evidence of a possible implication of Dpl has been recently produced by our group, reporting an aberrant $P R N D$ expression in the astrocytic and leukaemic specimens with different malignancy grades $(22,23)$.

In this study, we present the results of a data mining algorithm analysis of the PRND gene expression in a panel of astrocytic tumor specimens, performed to determine if $P R N D$ gene expression profiling can be used to generate a molecular classification of astrocytomas that potentially stratifies patients with respect to clinical data, such as the tumor grade of malignancy, the recurrence and the anatomical localization of the tumor.

\section{Materials and methods}

Tissue samples. A total of 111 patients with malignant astrocytomas were included in this study. All patients underwent surgery at Azienda Ospedaliera - Universitaria of Parma (Parma, Italy) and Azienda Ospedaliera - Universitaria of Verona (Verona, Italy). Tumor samples were evaluated by neuropathologists to confirm the diagnosis and were graded using the World Health Organization (WHO) criteria (2): six tumors were classified as pilocytic astrocytomas (WHO grade I), 15 as low-grade astrocytoma (II), 26 as anaplastic astrocytoma (III) and 64 as glioblastoma multiforme (IV). Among them, the majority (92/111) were primary or de novo tumors; 19 were referred to as secondary tumors. Patient characteristics, i.e. age, gender, tumor anatomical localisation, diagnosis and tumor recurrence are presented in Table I. Immediately after surgery, the samples were snap-frozen and stored at $-80^{\circ} \mathrm{C}$ until RNA extraction. As a negative control, total RNA from healthy adult human brain, pooled RNA from tissues of 70 trauma patients, purchased from Clontech (Palo Alto, CA), was adopted.

An avidin-biotin immunoperoxidase technique has been used to perform Ki-67 cytoproliferative indexes, using a monoclonal antibody (MIB-1, DakoCytomation, Glostrup, Denmark; dilution 1:80). For the evaluation, tumor areas with a high density of labelling were chosen. In five high power fields, a total of 500 cells were counted excluding the inflammatory and vessel cells. In these cells, the staining for the antigen was evaluated independently from their staining intensity and expressed as a percentage of immunoreactive cells.

Quantitative real-time RT-PCR. Total RNA from homogenised specimens was isolated with Trizol reagent (Invitrogen, Paisley,
Table I. Patient characteristics.

\begin{tabular}{|c|c|c|}
\hline & $\mathrm{n}$ & $\%$ \\
\hline \multicolumn{3}{|l|}{ Gender } \\
\hline Male & 63 & 56.8 \\
\hline Female & 48 & 43.2 \\
\hline Total & 111 & \\
\hline \multicolumn{3}{|l|}{ Age (years) } \\
\hline Median & 50.1 & \\
\hline Range & $6-80$ & \\
\hline \multicolumn{3}{|l|}{ Tumor location } \\
\hline Frontal & 31 & 27.9 \\
\hline Parietal & 14 & 12.6 \\
\hline Fronto/temporal & 4 & 3.6 \\
\hline Midbrain & 4 & 3.6 \\
\hline Brainstem & 10 & 9.1 \\
\hline Neofrontal & 1 & 0.9 \\
\hline Thalamus & 4 & 3.6 \\
\hline Temporal/occipital & 1 & 0.9 \\
\hline Occipital & 4 & 3.6 \\
\hline Temporal & 28 & 25.2 \\
\hline Cerebellum & 4 & 3.6 \\
\hline Basal ganglia & 4 & 3.6 \\
\hline Hippocampus & 1 & 0.9 \\
\hline Parietal/occipital & 1 & 0.9 \\
\hline \multicolumn{3}{|l|}{ WHO grading } \\
\hline I Pilocytic astrocytoma & 6 & 5.4 \\
\hline II Low-grade astrocytoma & 15 & 13.5 \\
\hline III Anaplastic astrocytoma & 26 & 23.4 \\
\hline IV Glioblastoma multiforme & 64 & 57.7 \\
\hline \multicolumn{3}{|l|}{ Tumor recurrence } \\
\hline Primary & 92 & 82.9 \\
\hline $\mathrm{I}$ & 4 & 3.6 \\
\hline II & 15 & 13.5 \\
\hline III & 18 & 16.2 \\
\hline IV & 55 & 49.6 \\
\hline Secondary & 19 & 17.1 \\
\hline $\mathrm{I}$ & 2 & 1.8 \\
\hline II & 0 & 0 \\
\hline III & 8 & 7.2 \\
\hline IV & 9 & 8.1 \\
\hline
\end{tabular}

Main clinical data of the analysed glioma patients are reported, such as gender, age, tumor location, WHO grading and recurrence.

UK) using the manufacturer's specification and accurately quantified using replicas and serial dilutions by means of the Nanodrop ND-1000 spectrophotometer (Nanodrop Technologies, Wilmington, DE). 


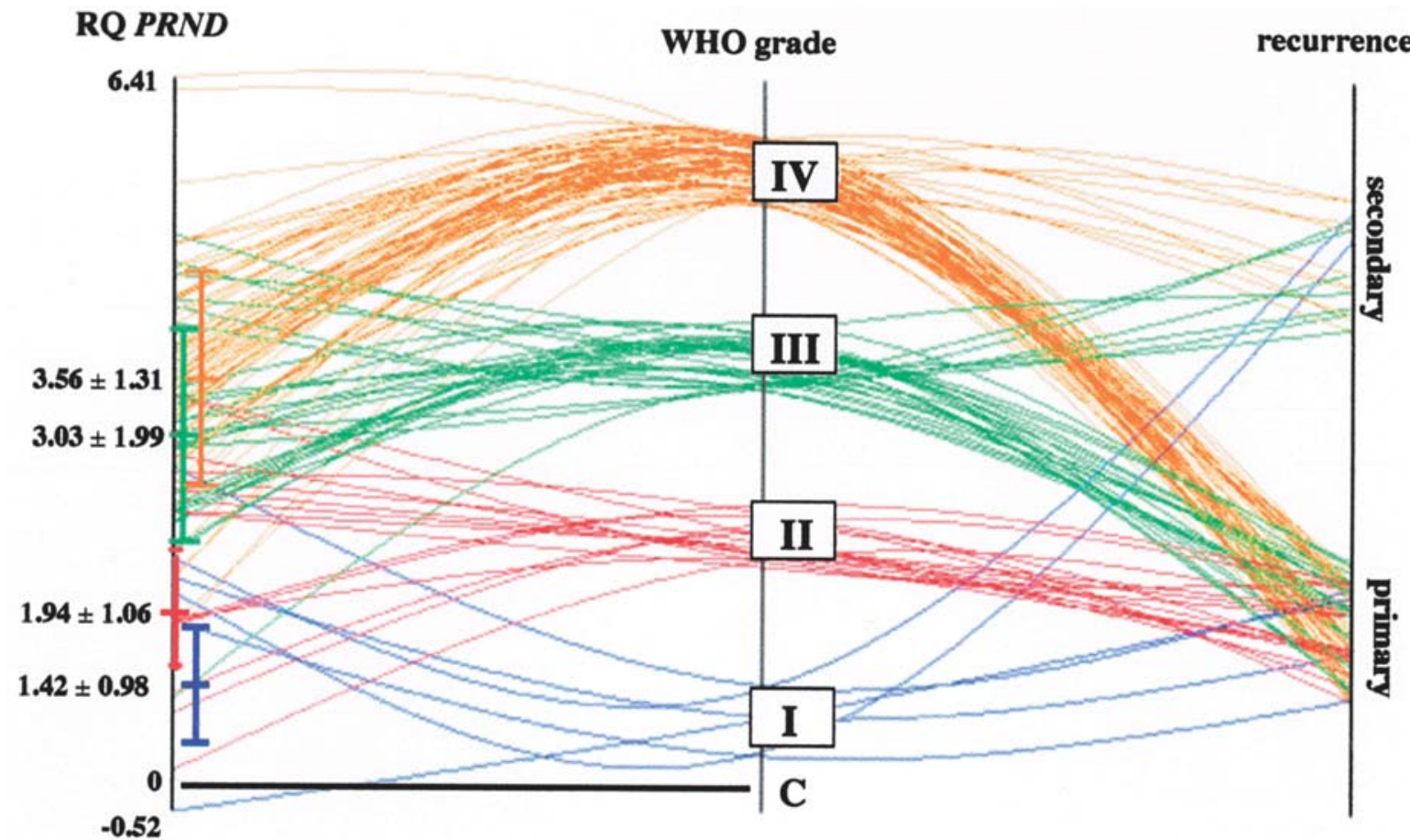

Figure 1. Parallel coordinates analysis of relative PRND expression (RQ PRND), WHO grades of malignancy (I, II, III, IV) and recurrence (primary or secondary) of the astrocytoma specimens. C, healthy brain control; median and standard deviations within each WHO grade are indicated with vertical bars.

Five $\mu \mathrm{g}$ of RNA of each sample was reverse-transcribed using the High Capacity cDNA Archive kit (Applera, Foster City, CA) according to the supplier's specifications. Genespecific Taqman Minor Groove Binders (MGB) probe and primers were designed by means of the Primer Express, version 3.1, software (Applera). Primers were designed on adjacent exons of the genes in order to avoid DNA-derived amplicons, as follows: PRND (Genbank accession number NM_012409), forward primer, 5'-ACTCCAGAGAGAGCCAAGGTTCT; reverse, 5'-GCCAGCCACCACCAGCT; probe: 5' 6-FAMCGATGAGGAAGCACC-MGB; a GAPDH Taqman probe for expression assays was purchased from Applera and used as reference. Real-time PCR was performed on an MJ Opticon II model (MJ Research, Waltham, MA) using 750 ng of RNA for each of the investigated samples. Samples were subjected to 40 amplification cycles of $95^{\circ} \mathrm{C}$ for $10 \mathrm{~min}$ and $59^{\circ} \mathrm{C}$ for $1 \mathrm{~min}$. For each sample, amplifications were performed in $50-\mu 1$ volumes containing the primers (900 $\mathrm{nM}$ each) and the probe $(200 \mathrm{nM}), 1 \mathrm{X}$ Universal PCR Master mix No Amperase UNG (Applera). RNA normalisation was performed using $G A P D H$ as a housekeeping gene, after a selection of appropriate reference genes (GAPDH, ACTB, $\beta$-tubulin, $H P R T)$. The $\mathrm{Ct}$ averages of the replicas performed for the genes were determined; then, PRND $\triangle \mathrm{Ct}\left(\mathrm{Ct}_{\text {PRND }}-\mathrm{Ct}_{\text {GAPDH }}\right)$ was calculated for each sample. As a validation test, plots of the log RNA dilutions versus $\triangle \mathrm{Ct}$ were made, showing that the amplification efficiencies of the target (PRND) and reference $(G A P D H)$ were similar (slopes: 0.0596 and 0.0605 , respectively). These validation experiments enabled the use of the ' $\triangle \Delta \mathrm{Ct}$ comparative method' (24) for relative quantitation of $P R N D$ expression. $P R N D$ relative expression (RQ PRND) was given as a result of the $\log$ of the relative expression, using the pooled normal brain healthy cDNAs as an internal calibrator. Astrocytoma samples were analysed in a blind-trail fashion and all experiments were performed in duplicate, with good consistency of the results (mean coefficient of variation was $3.2 \%$ ).

Statistics. The Orange data mining software (freely distributed under GPL at www.ailab.si/orange) was used to compare the $P R N D$ expression in the different tumor categories. In particular, for the nomograms and the hierarchical clustering analyses, a Naive Bayes learner/classifier and an Euclidean distance metric were adopted, respectively. ANOVA one-way and $\chi^{2}$ analyses with a confidence interval of $95 \%$ were used to correlate $P R N D$ expression with low versus high tumor grade specimens and within WHO grades of malignancy.

\section{Results}

PRND gene expression analysis. Table I shows the clinical characteristics of the 111 patients included in the present study. All patients (63 males and 48 females, ratio 1.3:1) were Italian. Their median age was 50.1 years (range, 6-80 years).

Total RNA was extracted from a panel of 111 astrocytoma specimens, previously histologically classified into WHO grades. RNA of each sample was accurately quantified using the Nanodrop ND-1000 spectrophotometer and $5 \mu \mathrm{g}$ was retrotranscribed into cDNA molecules. Different housekeeping genes (HPRT, ACTB, GAPDH, $\beta$-tubulin) were assayed for constitutive expression using two replicas of different dilutions of the cDNA samples (10-50 ng). Among them, GAPDH showed lesser variation in expression (standard deviation $(\mathrm{sd})=0.03)$, comparing to HPRT $(\mathrm{sd}=0.51), A C T B(\mathrm{sd}=0.26)$ and $\beta$-tubulin $(\mathrm{sd}=1.05)$. 

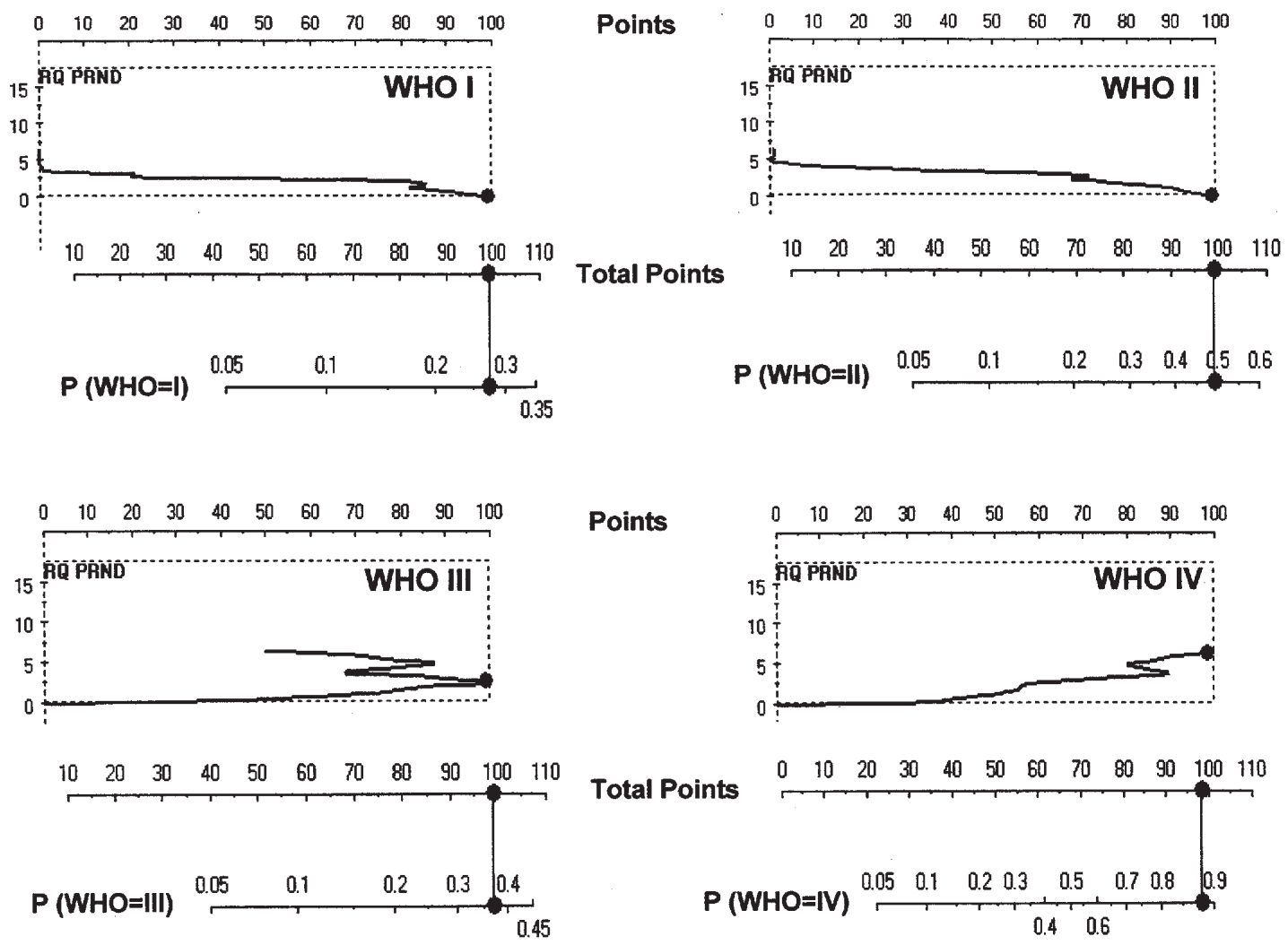

Figure 2. Nomogram analysis of relative PRND expression (RQ PRND) within the different WHO grades of malignancy (I, II, III, IV) of the astrocytoma specimens. Points and total points axes indicate the points attributed to each variable value and the sum of the points for each variable, respectively. $\mathrm{P}$ axes indicate the predicted probability that relates RQ PRND to each WHO grade.

Then, a Taqman MGB probe and gene-specific oligonucleotides were employed in order to amplify adjacent exons of the human PRND gene. cDNA (750 ng) was amplified using two independent replicates. PRND expression values were then normalised using $G A P D H$, as previously reported $(22,23)$. Differently from $G A P D H, P R N D$ relative expression (RQ $P R N D$ ) was highly variable between the astrocytoma specimens, ranging from -0.52 to $6.41 \mathrm{Logs}$ of expression, compared to the healthy brain sample. In particular, in agreement with previous evidence (22), the highest expression was detected in glioblastoma multiforme (WHO grade IV, RQ $P R N D=6.41$ ), while the lowest was in pilocytic astrocytomas (WHO grade I, RQ $P R N D=-0.52$ ) and in healthy control total brain samples (RQ $P R N D=0$ ). As reported in the parallel coordinates analysis (Fig. 1), low grades (WHO I and II) and negative control samples showed a different and statistically significant distribution compared to the high grades (WHO III and IV), according to PRND RQ values ( $\mathrm{P}<0.001$, ANOVA one-way). In the same graphical representation, the recurrence (primary or secondary) of the tumor specimens was reported, indicating that secondary type $(19 / 111,17.1 \%$ of total cases) occurred mainly in WHO grades III $(8 / 19,42.1 \%)$ and IV $(9 / 19,47.4 \%)$ and, noticeably, in two pilocytic astrocytoma WHO grade I specimens $(2 / 19,10.5 \%)$.

$P R N D$ relative expression was next subjected to a Naive Bayesian Nomogram analysis (25). In this analysis, reported in Fig. 2, RQ PRND values and probability plots were compared within the different WHO malignancy grades of the specimens. $P R N D$ expression profiles of the WHO I and II specimens were

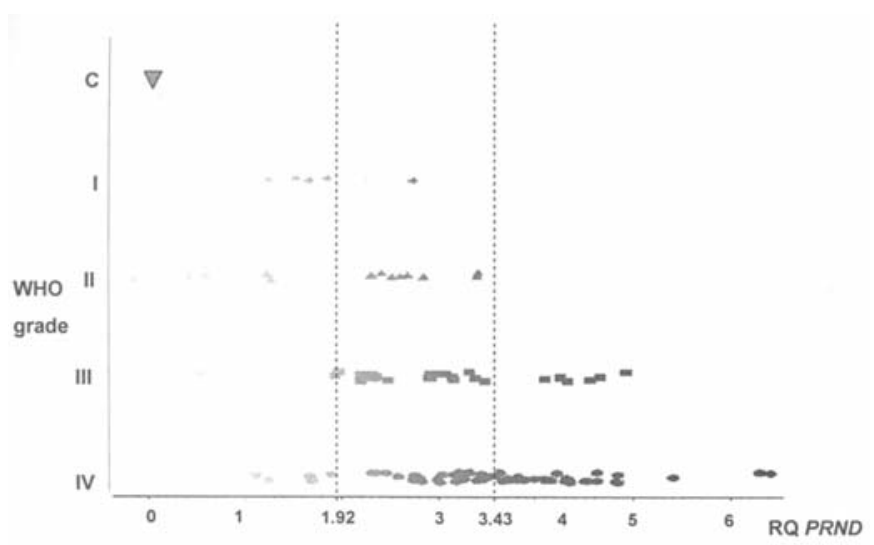

Figure 3. Scatterplot analysis of relative PRND expression (RQ PRND) distribution compared to the different WHO grades of malignancy (I, II, III, IV) of the astrocytoma specimens. C, healthy brain control. Three intervals of RQ PRND are defined by vertical dashed lines $\left(\mathrm{x}_{1}=1.92 ; \mathrm{x}_{2}=3.43\right)$. These define three $P R N D$ expression intervals, i.e. low- (RQ $P R N D<1.92)$, medium$(1.93<\mathrm{RQ} P R N D<3.42)$ and high-PRND expression (RQ $P R N D>3.43)$.

nearly monotonic and congruent, with higher scores (total points) in correspondence to low $P R N D$ expression; in contrast, $P R N D$ expression profiles within WHO III and IV specimens exhibited some differences, showing a monotonic-like trend for WHO grade IV and a more complex PRND expression curve for grade III samples. In order to better differentiate the specimens according to their grades of malignancy, a Scatterplot analysis was performed, adopting three specific 
Table II. Relative $P R N D$ expression intervals and WHO grading.

\begin{tabular}{|c|c|c|c|c|c|c|}
\hline \multirow[b]{3}{*}{ RQ PRND } & & \multicolumn{4}{|c|}{ WHO grades } & \multirow[b]{3}{*}{ Total } \\
\hline & & \multicolumn{2}{|c|}{ Low } & \multicolumn{2}{|c|}{ High } & \\
\hline & & I & $\overline{\text { II }}$ & III & IV & \\
\hline Low & $<1.92$ & 5 & 6 & 2 & 5 & 18 \\
\hline Medium & $1.93-3.42$ & 1 & 9 & $18^{\mathrm{a}}$ & 19 & 47 \\
\hline High & $>3.43$ & 0 & 0 & $6^{\mathrm{a}}$ & 40 & 46 \\
\hline Total & & 6 & 15 & 26 & 64 & 111 \\
\hline
\end{tabular}

RQ PRND intervals of expression in the glioma specimens according to their WHO grade of malignancy. ${ }^{\mathrm{a}} \mathrm{P}<0.001$ ( $\chi^{2}$ test) versus WHO grade IV.

intervals of $P R N D$ expression (Fig. 3): these intervals, as reported in Table II, better differentiate the specimens into their WHO grades of malignancy. According to this analysis, pilocytic astrocytoma specimens mainly exhibited low $P R N D$ expression values $(5 / 6,83.3 \%$ of grade I cases), while glioblastoma multiforme specimens had mainly high PRND expression $(40 / 64,62.5 \%)$; grade II and III samples mainly showed medium PRND expression (9/15, 60.1\%; and 18/26, $69.2 \%$, respectively). In particular, the distribution of grade III was significantly different compared to grade IV specimens $\left(\mathrm{P}<0.001, \chi^{2}\right.$ test $)$.

We then performed hierarchical clustering, a standard unsupervised learning method (26) of the tumor specimens. The main clinical information, such as the anatomical localization, age, gender, WHO grading, cytoproliferative index and tumor recurrence, were simultaneously compared to the PRND expression values, in order to identify homogeneous clusters. As reported in the dendrogram of Fig. 4, different groups of samples were created, according to the above mentioned criteria. The samples were roughly subdivided into their WHO grades of malignancy. The negative control sample was clustered with a cerebellar pilocytic astrocytoma specimen and this cluster was related to the low-grade specimens, as well as with anaplastic astrocytoma specimens expressing medium levels of $P R N D$ transcripts. Secondary-derived tumors were separated from primary; in particular, the two recurrent grade I samples were clustered together (indicated at the bottom of Fig. 4), showing very similar PRND expression values (RQ $P R N D=1.54$ and 1.86 , respectively), but having different anatomical localization (frontal cortex and thalamus). In the dendrogram with the primary-derived specimens, in correspondence to the most represented anatomical areas, i.e. frontal $(n=31)$ and temporal cortex $(n=28)$, three different subsets of the specimens were identified. In particular, within the frontal cluster, two subsets were highlighted: the former, subset A in Fig. 4, composed of grade IV specimens showed medium/high PRND expression values (RQ PRND median, 4.01; range, 2.30-6.41) and high cytoproliferative indexes (median Ki-67, 30.6; range, 10.2-42.6) and the latter, subset B, composed of grade II samples, having low/medium PRND expression values (RQ PRND median, 1.93; range, 0.413.40) and with lower cytoproliferative indexes (median Ki-67, 4.8; range, 0.8-6.1). Within the temporal-derived specimens, the grade IV subset $\mathrm{C}$ with medium/high $P R N D$ expression (RQ PRND median, 3.54; range, 2.58-4.30) and with high cytoproliferative indexes (median Ki-67, 22.1; range, 3.143.5) was highlighted. A significant difference in Ki-67 values $(\mathrm{p}<0.05$, one-way ANOVA) was found between low- (median, 2.4; range, 0.3-10.7) and high-grade astrocytomas (median, 18.5; range, 1.2-42.6), according to previous reports $(27,28)$. As expected, the gender and age of the patients provided no additional information to the classifying tree (data not shown).

\section{Discussion}

The new challenge in cancer biology is to move from purely morphological classification of tumors to one that is based on the integration of histological and molecular criteria (29). Particularly, in the light of the development of new pharmacological treatments, the identification of patient subsets with specific molecular signatures within tumor malignancy grades is becoming more and more relevant (29). In addition, molecular classification studies are likely to identify novel, clinically-relevant astrocytoma subsets that warrant additional detailed investigation (30-32).

In particular, our laboratory has previously identified a novel promising glioma marker, the doppel gene $(P R N D)$, which is overexpressed in high malignant astrocytomas, compared with normal brain tissue, showing barely detectable levels (22). To approach some of the molecular reappraisal goals in astrocytoma classification, we measured by real-time PCR the PRND mRNA levels in tumor specimens from a large cohort of 111 patients with malignant astrocytomas, histologically classified according to the WHO criteria. Firstly, an accurate RNA quantitation was performed using replicas and serial dilutions with a high sensitive Nanodrop ND-1000 spectrophotometric instrument, designed for measuring nucleic acid concentrations in sample volumes of 1-2 microliters: this was particularly important in the case of some pilocytic astrocytoma specimens, where the bioptical resections were particularly reduced. Then, a comparison of the expression of housekeeping gene transcripts such as glyceraldehyde-3phosphate dehydrogenase $(G A P D H), \beta$-actin $(A C T B), \beta$ tubulin and hypoxantine phosphoribosyltransferase (HRPT) was performed. As a result, GAPDH expression appeared nearly constant, compared with the other genes. Additionally, the less variable GAPDH expression levels were in accordance with previous real-time PCR analysis on tumor-derived specimens $(22,23)$.

A quantitative relative real-time analysis of $P R N D$ expression, using a normal brain control (cDNA pooled from brains of 70 non-tumoral adults) showed a progressive increase in the $P R N D$ detection rate with increasing grade of astrocytoma malignancy. These data are in agreement with our previously reported data in astrocytomas (22), as well as in different tumors, such as those of haematologic origin (23). The level of PRND mRNA estimated with real-time RT-PCR procedure is the average amount of transcripts in a whole tumor sample and mainly depends on the number of $P R N D$-positive cells present in the tumor tissue. To this regard, 


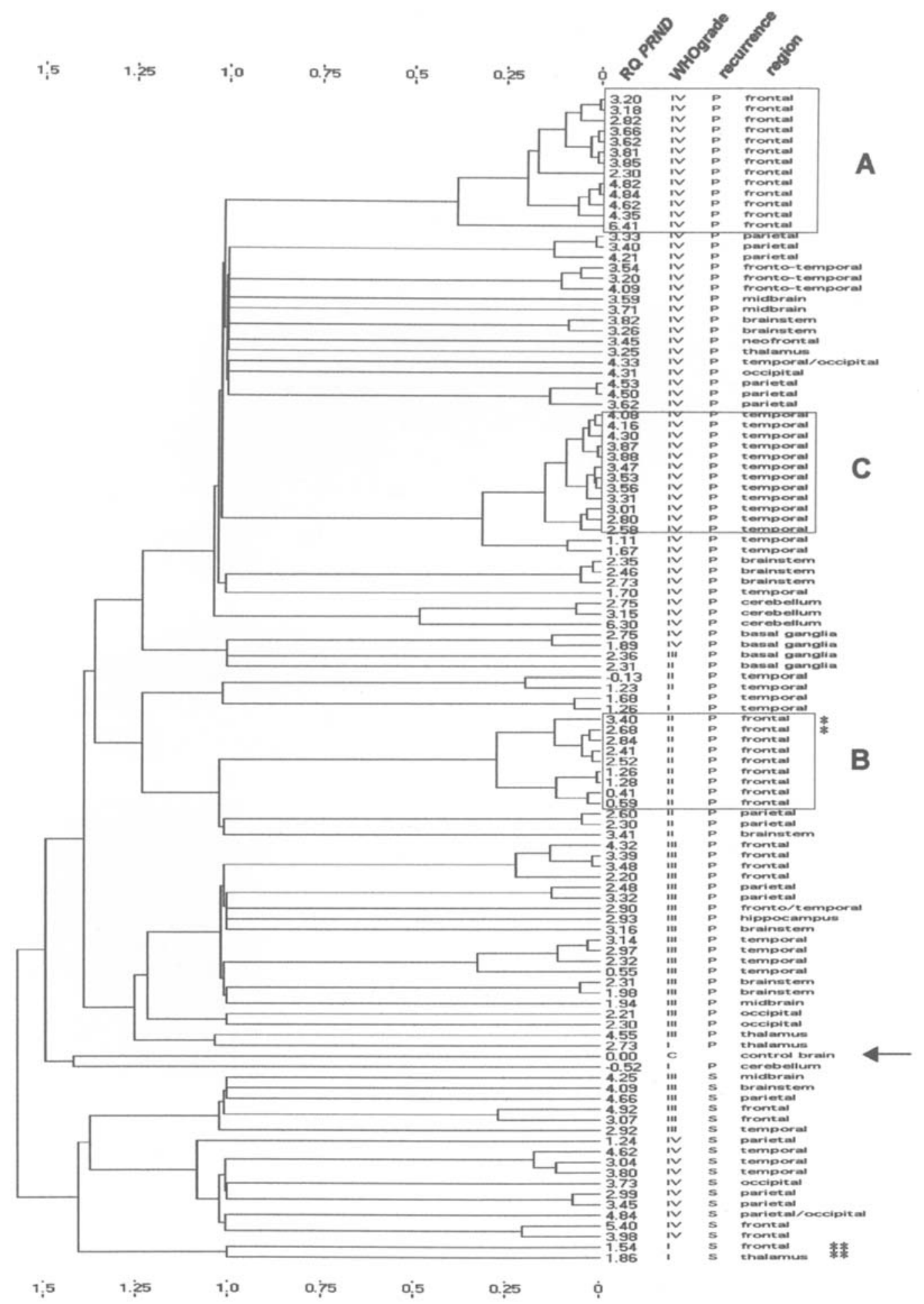

Figure 4. Dendrogram comparing PRND expression (RQ PRND), WHO grades of malignancy (I, II, III, IV), recurrence (primary or secondary) and anatomical localisation of the tumor specimens. WHO grade IV subset (A), with medium/high PRND expression values (see Results), high cytoproliferative indexes and a WHO grade II subset (B), with low/medium PRND expression values and lower cytoproliferative indexes, both localised at the frontal cortex are indicated. A third temporal-localised grade IV subset (C) with medium/high PRND expression and high cytoproliferative indexes is reported. The healthy brain control (C) is indicated with an arrow. Tumor recurrence is indicated by P (primary) or S (secondary). "Two previously identified low-grade astrocytoma specimens, classified as mixed oligoastrocytomas after histologic re-examination. ${ }^{* *}$ Two recurrent grade I samples, described in the text.

studies of PRND mRNA in situ hybridization (33) and Dpl immunohistochemistry (22) have been performed, indicating that within the tumor area, the astrocytic neoplastic cells mainly exhibited increase in $P R N D$ expression. However, the reasons for doppel mRNA overexpression in astrocytic tumor cells are unknown. Unfortunately, very little is known, at present, about the physiological functions of the doppel gene; even less information is available about its possible pathological role. To this regard, the up-regulation of astrocyte-specific genes in prion-deficient mice, ectopically expressing doppel, has suggested that doppel was actively involved in glial cell activation in the brain (34). 
In the examined cohort of astrocytoma patients, PRND showed two opposite monotonic-like expression profiles in correspondence to low-grade astrocytomas (WHO I and II) and glioblastoma multiforme (WHO IV), as evidenced in the nomogram analysis. In contrast, the PRND expression profile in anaplastic astrocytoma (WHO III) shared some of the previously indicated profiles, suggesting a sort of transition of $P R N D$ expression between low and high grades of malignancy. These results reinforced the concept that anaplastic astrocytomas are intermediate-grade tumors, showing detectable mitotic activity (absent in low-grade astrocytomas) but not necrosis and prominent vascular proliferation, characteristic of glioblastoma multiforme $(3,12)$. The distribution of the specimens according to their levels of $P R N D$ expression, as exemplified in the analysis, did not suggest per se an exact subdivision into the different four grades of astrocytoma malignancy; however, low- and highgrade astrocytomas were clearly differentiated $(\mathrm{P}<0.001)$. Noticeably, in the case of high-grade astrocytomas, $P R N D$ expression may represent an additional diagnostic indicator for grade III/IV uncertain classification. The PRND gene expression profiling was also used to identify novel subsets of astrocytomas, using a combination of unsupervised and supervised algorithms. We have identified different subsets, biologically and clinically different, in terms of recurrence, malignancy, cellular proliferation and anatomical localization of the tumor. Globally, there are limitations to the present study; in particular, the use of supervised approaches, based on the assumption that the grouping (i.e. the histological tumor diagnoses) is correct, may not be a valid assumption for all the clinical cases examined. In particular, it was evident that astrocytoma patients formed continuous PRND expression, also some low-grade specimens were located near to the glioblastoma multiforme clusters. In these cases, for example, the review of the clinical data upon histologic re-examination, for two histologically classified grade II specimens, located in the frontal cluster of the PRND expression dendrogram, showed morphological features suggestive of possible astrocytic differentiation into mixed oligoastrocytomas. Therefore, it is possible that the differentiation processes that accomplished these cellular transformations could in part support the relatively high $P R N D$ expression values (RQ $P R N D=3.4 \mathrm{O}$ and 2.68, respectively). Globally, the hierarchically clustering analysis showed that tumor classification based even on a single-gene quantitation could generate a patient stratification that could be clinically relevant and more informative than a single conventional histological classification.

In conclusion, our findings suggest that the level of PRND mRNA may be used as an indicator of enhanced astrocytoma malignancy. More comprehensive gene-expression models, joining $P R N D$ profiles with other tumor-markers, may therefore complement histology and help to refine a finer tumor grading and classification.

\section{Acknowledgments}

Orange Software is released under General Programming License (GPL). We are therefore particularly grateful to Orange Program's authors, Demsar J, Zupan B, Leban G: (2004) Orange: From Experimental Machine Learning to Interactive
Data Mining, White Paper (www.ailab.si/orange), Faculty of Computer and Information Science, University of Ljubljana (Slovenia). The authors are also grateful to Professor Andrea Mattevi (University of Pavia, Italy) for providing the Nanodrop ND-1000 spectrophotometer. This study was supported by grants from the Italian MIUR, 'Progetti di Ricerca di Rilevante Interesse Nazionale (2005)'.

\section{References}

1. Mikkelsen T, Cairncross JG and Cavenee WK: Genetics of the malignant progression of astrocytoma. J Cell Biochem 46: 3-8, 1991.

2. Kleihues $P$ and Cavenee WK: World Health Organization Classification of Tumors of the Nervous System. Lyon, 2000.

3. Ichimura K, Ohgaki H, Kleihues P and Collins VP: Molecular pathogenesis of astrocytic tumours. J Neurooncol 70: 137-160, 2004.

4. Vogelstein B and Kinzler KW: The multistep nature of cancer. Trends Genet 9: 138-141, 1993.

5. Sehgal A: Molecular changes during the genesis of human gliomas. Semin Surg Oncol 14: 3-12, 1998.

6. Zhu Y and Parada LF: The molecular and genetic basis of neurological tumours. Nat Rev Cancer 2: 616-626, 2002.

7. Fuller GN, Rhee CH, Hess KR, Caskey LS, Wang R, Bruner JM, Yung WK and Zhang W: Reactivation of insulin-like growth factor binding protein 2 expression in glioblastoma multiforme: a revelation by parallel gene expression profiling. Cancer Res 59: 4228-4232, 1999.

8. Sallinen SL, Sallinen PK, Haapasalo HK, Helin HJ, Helen PT, Schraml P, Kallioniemi OP and Kononen J: Identification of differentially expressed genes in human gliomas by DNA microarray and tissue chip techniques. Cancer Res 60: 6617-6622, 2000.

9. Rickman DS, Bobek MP, Misek DE, Kuick R, Blaivas M, Kurnit DM, Taylor J and Hanash SM: Distinctive molecular profiles of high-grade and low-grade gliomas based on oligonucleotide microarray analysis. Cancer Res 61: 6885-6891, 2001.

10. Godard S, Getz G, Delorenzi M, Farmer P, Kobayashi H, Desbaillets I, Nozaki M, Diserens AC, Hamou MF, Dietrich PY, Regli L and Janzer RC: Classification of human astrocytic gliomas on the basis of gene expression: a correlated group of genes with angiogenic activity emerges as a strong predictor of subtypes. Cancer Res 63: 6613-6625, 2003.

11. Ohgaki H: Genetic pathways to glioblastomas. Neuropathology 25: 1-7, 2005.

12. Rasheed BK, Wiltshire RN, Bigner SH and Bigner DD: Molecular pathogenesis of malignant gliomas. Curr Opin Oncol 11: 162-167, 1999.

13. Comincini S: Searching for molecular markers of human gliomas. Funct Neurol 16: 291-298, 2001.

14. Moore RC, Lee IY, Silverman GL, Harrison PM, Strome R, Heinrich C, Karunaratne A, Pasternak SH, Chishti MA, Liang Y, Mastrangelo $\mathrm{P}$ and Wang $\mathrm{K}$ : Ataxia in prion protein (PrP)deficient mice is associated with upregulation of the novel PrPlike protein doppel. J Mol Biol 292: 797-817, 1999.

15. Comincini S, Foti MG, Tranulis MA, Hills D, Di Guardo G, Vaccari G, Williams JL, Harbitz I and Ferretti L: Genomic organization, comparative analysis, and genetic polymorphisms of the bovine and ovine prion Doppel genes (PRND). Mamm Genome 12: 729-733, 2001.

16. Makrinou E, Collinge J and Antoniou M: Genomic characterization of the human prion protein (PrP) gene locus. Mamm Genome 13: 696-703, 2002.

17. Behrens A: Physiological and pathological functions of the prion protein homologue Dpl. Br Med Bull 66: 35-42, 2003.

18. Mastrangelo $\mathrm{P}$ and Westaway $\mathrm{D}$ : The prion gene complex encoding $\operatorname{PrP}(\mathrm{C})$ and Doppel: insights from mutational analysis. Gene 275: 1-18, 2002.

19. Behrens A and Aguzzi A: Small is not beautiful: antagonizing functions for the prion protein $\operatorname{Pr} P(C)$ and its homologue Dpl. Trends Neurosci 25: 150-154, 2002.

20. Tuzi NL, Gall E, Melton D and Manson JC: Expression of doppel in the CNS of mice does not modulate transmissible spongiform encephalopathy disease. J Gen Virol 83: 705-711, 2002 . 
21. Ferrer I, Freixas M, Blanco R, Carmona M and Puig B: Selective PrP-like protein, doppel immunoreactivity in dystrophic neurites of senile plaques in Alzheimer's disease. Neuropathol Appl Neurobiol 30: 329-337, 2004.

22. Comincini S, Facoetti A, Del Vecchio I, Peoc'h K, Laplanche JL, Magrassi L, Ceroni M, Ferretti L and Nano R: Differential expression of the prion-like protein doppel gene (PRND) in astrocytomas: a new molecular marker potentially involved in tumor progression. Anticancer Res 24: 1507-1517, 2004.

23. Travaglino E, Comincini S, Benatti C, Azzalin A, Nano R, Rosti V, Ferretti L and Invernizzi R: Overexpression of the Doppel protein in acute myeloid leukaemias and myelodysplastic syndromes. Br J Haematol 128: 877-884, 2005.

24. Livak KJ and Schmittgen TD: Analysis of relative gene expression data using real-time quantitative PCR and the 2(-Delta Delta C(T)) method. Methods 25: 402-408, 2001.

25. Partin AW, Yoo J, Carter HB, Pearson JD, Chan DW, Epstein JI and Walsh PC: The use of prostate specific antigen, clinical stage and Gleason score to predict pathological stage in men with localized prostate cancer. J Urol 150: 110-114, 1993.

26. Hastie T, Tibshirani R, Botstein D and Brown P: Supervised harvesting of expression trees. Genome Biol 2: RESEARCH0003. Epub Jan 10, 2001.

27. Torp SH: Diagnostic and prognostic role of Ki67 immunostaining in human astrocytomas using four different antibodies. Clin Neuropathol 21: 252-257, 2002

28. Kayaselcuk F, Zorludemir S, Bal N, Erdogan B, Erdogan S and Erman T: The expression of survivin and Ki-67 in meningiomas: correlation with grade and clinical outcome. J Neurooncol 67: 209-214, 2004.
29. Louis DN, Holland EC and Cairncross JG: Glioma classification: a molecular reappraisal. Am J Pathol 159: 779-786, 2001.

30. Fuller GN, Hess KR, Rhee CH, Yung WK, Sawaya RA, Bruner JM and Zhang W: Molecular classification of human diffuse gliomas by multidimensional scaling analysis of gene expression profiles parallels morphology-based classification, correlates with survival, and reveals clinically-relevant novel glioma subsets. Brain Pathol 12: 108-116, 2002.

31. Mischel PS, Shai R, Shi T, Horvath S, Lu KV, Choe G, Seligson D, Kremen TJ, Palotie A, Liau LM, Cloughesy TF and Nelson SF: Identification of molecular subtypes of glioblastoma by gene expression profiling. Oncogene 22: 2361-2373, 2003.

32. Liang Y, Diehn M, Watson N, Bollen AW, Aldape KD, Nicholas MK, Lamborn KR, Berger MS, Botstein D, Brown PO and Israel MA: Gene expression profiling reveals molecularly and clinically distinct subtypes of glioblastoma multiforme. Proc Natl Acad Sci USA 102: 5814-5819, 2005.

33. Comincini S, Chiarelli LR, Zelini P, Del Vecchio I, Azzalin A, Arias A, Ferrara V, Rognoni P, Dipoto A, Nano R, Valentini G and Ferretti L: Nuclear mRNA retention and aberrant doppel protein expression in human astrocytic tumor cells. Oncol Rep 16: 1325-1332, 2006.

34. Atarashi R, Sakaguchi S, Shigematsu K, Arima K, Okimura N, Yamaguchi N, Li A, Kopacecek J and Katamine S: Abnormal activation of glial cells in the brains of prion protein deficient mice ectopically expressing prion protein-like protein PrPLP/Dpl. Mol Med 7: 803-809, 2001 